\title{
The Influence of Wettability on the Droplet Impact onto Micro-Structured Surfaces
}

\author{
Daniela Ribeiro*1, Patrick Foltyn², André Silva ${ }^{1}$, Grazia Lamanna ${ }^{2}$, Bernhard Weigand $^{2}$ \\ ${ }^{1}$ University of Beira Interior, AEROG-LAETA, Covilhã, Portugal \\ ${ }^{2}$ University of Stuttgart, Institute for Aerospace Thermodynamics (ITLR), Stuttgart, Germany \\ ${ }^{*}$ Corresponding author email: daniela.santo.ribeiro@ubi.pt
}

\begin{abstract}
The flourishing of applications in need of self-cleaning mechanisms increased the search for water repellent hydrophobic surfaces with induced roughness. Disclosing the small-scale interface phenomena on the wetting behavior is essential to design efficient hydrophobic materials with defined topography. On the other hand, the spreading behavior concerning the formation of thin films on a surface is required to assure the quality of spray cooling and coatings. The contact angle undoubtedly plays an important role in the droplet impact, providing different outcomes. Moreover, an open question is, how surface topography can affect the impact process. Therefore, to evaluate these matters, different surface patterns were manufactured to assess the surface topography influence on the impact dynamic behavior. Additionally, the wettability of the micro-structured surfaces was flexibly influenced through plasma activation and plasma polymerization. The impact of distilled water and isopropanol droplets on the different surface patterns was captured from three perspectives providing high-quality images of the phenomena. Different surface morphologies can be obtained depending on the surface micro-structures and wettability, affecting spreading shape and evolution. The fluid penetration within the micro-structures is a key feature influencing not only the structures of the outcomes but also the transition between regimes.
\end{abstract}

\section{Keywords}

Micro-structured surfaces; hygrophilic; hygrophobic; drop impact; wettability

\section{Introduction}

The droplet impingement upon a dry surface is an essential phenomenon in multiple areas, from nature to technical applications [1]. Understanding the involved physical processes and predicting its behavior is pursued in a variety of research areas.

The ability of a fluid to spread on a dry solid surface is defined as wettability and is evaluated by the apparent contact angle, $\theta_{\text {app }}$. The wettability of a surface can range from full wetting to non-wetting. The surface is hygrophilic if the apparent contact angle between the liquid droplet and the solid surface is small, $\theta_{\text {app }}<90^{\circ}$. It is called hydrophilic if the fluid is water. This wetting behavior enhances heat transfer between the droplet and the wall. Hygrophobic surfaces exhibit a large apparent contact angle, $\theta_{\text {app }}>90^{\circ}$, so, a non-wetting behavior. There is a wide range of applications for this regarding the underlying self-cleaning mechanism known as "Lotus effect". The role of wettability is indispensable to design efficient surperhygrophobic materials $\left(\theta_{\text {app }}>150^{\circ}\right)$ [2]. The spreading dynamics are highly associated with wettability and the spreading rate increases while decreasing the contact angle [3]. However, for high Weber numbers, the effect of wettability seems to be reduced or even negligible [4].

A rough surface can be composed of regular micro-structures or random irregular asperities. Focusing on regular micro-structured surfaces, splashing can be inhibited by increasing the height of the structures which form the substrate [5]. On the other hand, splashing can be enhanced by altering the arrangement of the pillars that constitute the structured surfaces [6]. Additionally, the volume of entrapped air at the gaps within the structures can affect the intensity 
of splashing. Applying different arrangements and geometries to the surface micro-structures can even create directional splashing. For these complex surface topographies, mean roughness is not able to fully characterize the morphological behavior of the impact and additional length scales have to be considered to understand their influence on the impact outcome.

One of the most relevant questions concerns the wetting distribution along the surface. A heterogeneous wetting state is reached by the combined influence of large contact angles and surface roughness. Hensel et al. [7] stated that a heterogeneous wetting state can be achieved even for low surface tension fluids. It happens when air is entrapped inside the grooves underneath the liquid film and can be characterized by the Young contact angle, $\theta_{Y}$. This wetting state is usually achieved for water at $\theta_{Y}>90^{\circ}$ and an appropriate roughness [8]. The heterogeneous wetting state is energetically metastable and can reach a homogeneous wetting state through pressure fluctuations (acoustical or mechanical vibrations), resulting in a weak resistance to wetting. The hydrostatic pressure inside the cavity continuously increases. So, there is a critical pressure difference where the breakthrough scenario is achieved. There are two possible scenarios: the canthotaxis effect and the Laplace breakthrough [7]. For both cases, the breakthrough pressure is inversely proportional to the distance between the three-phase contact line and the symmetry center of the cavity.

It is well known that hygrophilic surfaces enhance droplet spreading, improving the heat transfer between the solid and the liquid. On the other hand, hygrophobic surfaces have self-cleaning properties. What if the effect of the wettability behavior is combined with surface topography? Questions arise regarding which parameter will be more dominant on the morphology impact, if certain wettabilities can overlap the effect of surface structure or if a particular micro-structured arrangement can enhance or not the wettability effect. Thus, the main goal of this study is to investigate the effect of wettability on single droplet impact onto micro-structured surfaces.

A wide range of impact conditions was defined, and several experiments were performed. Using Lexan ${ }^{\circledR}$ (Polycarbonate, PC) as surface material, four different micro-structured patterns were manufactured to be used as target surfaces. Distilled water and isopropanol droplets impinged on these surfaces from four different falling heights resulting into four distinct impact energies for each fluid. The Weber numbers range from 80 to $1,165, W e=\rho u_{0}^{2} D_{0} / \sigma$, and the Reynolds numbers from 1,135 to $12,240, R e=\rho D_{0} u_{0} / \mu$, where $\rho$ is the droplet fluid density, $\sigma$ the surface tension, $\mu$ the viscosity, $D_{0}$ the droplet impact diameter and $u_{0}$ the impact velocity. A wettability behavior from full wetting to non-wetting was obtained by applying plasma treatments to the micro-structured surfaces. From these experiments, different impact regimes were identified and mapped depending on the surface topography and wettability. The morphological behavior of the impact was captured from different perspectives, allowing the detailed investigation of the heterogeneous wetting state.

\section{Material and Methods Experimental Setup}

To record these experiments, a complex experimental setup was designed and built. Image acquisition system, triggering and synchronization unit, and droplet dispensing system are the main parts of this test rig. High-speed imaging of three different perspectives of the phenomena obtained for the droplet impact onto micro-structured surfaces was captured to enhance the knowledge about the droplet dynamic behavior. Lateral and top perspectives are acquired from diffuse backlight imaging and share the same CMOS-chip by using a beam splitter plate. Due to that, only two synchronized Photron SA-X2 high-speed cameras were needed to record the experiments from the three perspectives. The bottom view was recorded in a total internal reflection configuration, providing insight into the wetted and dry areas inside the gaps within the structure. The resolution of both cameras was set at $1,024 \times 672 \mathrm{px}^{2}$ with a shutter time of $1 / 88,888 \mathrm{~s}$ and a frame rate of $20,000 \mathrm{fps}$. Both cameras were synchronized and triggered when a droplet passes a LASER light barrier providing a TTL-signal. Finally, the droplet dispensing 


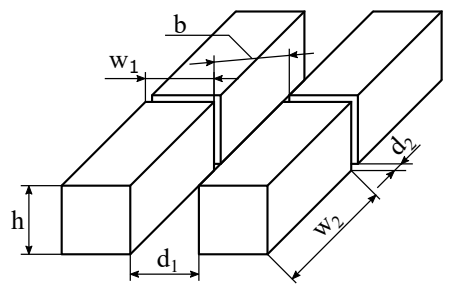

(a)

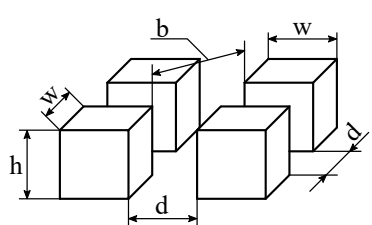

(b)

Figure 1. Dimensions of the micro-structured surfaces: a) grooves, $S 1$; b) square-cylindrical pillars, $S 2-S 4$.

system was composed of a medical syringe pump with a glass syringe connected to a needle by Teflon ${ }^{\circledR}$ tubing equipment. Glass and Teflon ${ }^{\circledR}$ were the materials chosen for the pumping system to avoid chemical interactions during the pumping process. The needle was tilted by $45^{\circ}$ to avoid a droplet detachment at different positions. The samples were placed on top of the glass prism that makes the bottom perspective possible and a film of isopropanol was placed in between the top surface of the prism and the surface sample to allow the total internal reflection to take place inside the polymeric surface sample.

\section{Micro-structured Surfaces and Wetting Behavior Modifications}

Lexan ${ }^{\circledR}$ (Polycarbonate, PC) surfaces with four different micro-structured patterns were manufactured to perform this study. Figure 1 shows the different patterns, which were composed of large grooves $(S 1)$ or square-cylindrical pillars $(S 2-S 4)$. The height of the micro-structures was kept constant at $h=20 \mu \mathrm{m}$. The micro-structures dimensions are shown in Table 1. $S 1$ is composed of very long pillars with a width of $w_{1}=60 \mu \mathrm{m}$ and a length of $w_{2}=500 \mu \mathrm{m}$, the orthogonal spacing between grooves is $d_{1}=60 \mu \mathrm{m}$, and the gap into the direction of the grooves is $d_{2}=15 \mu \mathrm{m}$, due to manufacturing constraints. The other surface patterns, $S 2-S 4$ are composed of square-cylindrical pillars with an edge length equal to the space between the pillars: $w=d=60 \mu \mathrm{m}$ for $S 2 ; w=d=30 \mu \mathrm{m}$ for $S 3 ; w=d=15 \mu \mathrm{m}$ for $S 4$. The surface specimens are manufactured by a combination of the LASER lithography, nickel electroforming and the hot embossing technique at Karlsruhe Nano and Micro Facility (KNMF), Germany [9]. Through a nickel shim, a master mold is produced with the negative of the structures. The reproduction method is very accurate and can reproduce constantly the final micro-structured samples at high quality. Small defects were first identified through quality control using Scanning Electron Microscopy (SEM) analysis and micro-Computed Tomography (microCT). During the experiments, the samples were investigated with an optical microscope after each set of impacts for quick and easy quality control where all defects were carefully mapped. This procedure was important to check and confirm the durability of the specimens since several droplets are impinging on the same sample and the impact velocity was incrementally increased. At the end of the experiments, no damages or changes on the micro-structures were detected, guaranteeing the long-term durability of the surfaces.

Different length scales can characterize micro-structured surfaces. The arithmetical mean roughness is one of the most commonly used in literature to define rough surfaces $R_{a}$ [10], defining the absolute deviation of roughness irregularities from the center line. The roughness factor $r$ was introduced by Wenzel [11] describes the ratio between the actual surface area and the projected surface area. For smooth surfaces $r=1$ and for rough surfaces $r>1$. Tsai et al. [6] defined some length scales regarding the arrangement of the pillars and $b$ is the diagonal between two elements, as shown in Figure 1. Due to the fact that all micro-structures have the same height, a larger $b$ corresponds to a larger distance between the three-phase contact line and the symmetry center of the cavity, which is inversely proportional to the smallest pressure difference to reach the breakthrough pressure. These length scales are described in Table 1. The wetting behavior of the liquid-solid system was modified using plasma activation and 
Table 1. Dimensions of the micro-structured surfaces for the different surface patterns.

\begin{tabular}{c|ccc|ccc}
\hline \multirow{2}{*}{ ID } & \multicolumn{3}{|c|}{ Dimensions $[\mu \mathrm{m}]$} & \multicolumn{4}{|c}{ Topography Parameters } \\
& Edge Length & Distance & Height & $R_{a}[\mu \mathrm{m}]$ & $r$ & $b[\mu \mathrm{m}]$ \\
\hline \multirow{2}{*}{$S 1$} & $w_{1}=60$, & $d_{1}=60$, & $h=20$ & 10 & 1.36 & 61.85 \\
$S 2$ & $w_{2}=500$ & $d_{2}=15$ & & & & \\
$S 3$ & $w=60$ & $d=60$ & $h=20$ & 7.5 & 1.33 & 84.85 \\
$S 4$ & $w=30$ & $d=30$ & $h=20$ & 7.5 & 1.67 & 42.43 \\
& $w=15$ & $d=15$ & $h=20$ & 7.5 & 2.33 & 21.21 \\
\hline
\end{tabular}

plasma polymerization. Plasma activation promotes the wetting behavior of water with polymers. During this process, free radicals replace some groups of the surface atomic structure. This treatment only allows the change of the wettability behavior between the Lexan ${ }^{\circledR}$ surfaces and distilled water since isopropanol already shows a full wetting behavior with these polymeric surfaces. Plasma polymerization decreases the wettability of a liquid-solid system. Due to these plasma treatments, the apparent contact angle with both, water and isopropanol, was increased, and the values are identified in Table 2.

\section{Experimental Procedure and Parameter Space}

To perform the experiments, a detailed procedure was established. A smooth surface sample was assigned as "partner sample" to each structured surface sample. For each wettability class and fluid, a separate pair of structured sample and partner sample was assigned, so that one sample always covers the same parameter setting. The smooth partner sample was used for obtaining the surface wettability due to several reasons. First, contact angle measurements on structured surfaces are very complex and a contact angle distribution depending on the angle of observation needs to be measured [9]. Second, for activated surface samples, the measurement of the wetting behavior would already start to deactivate the sample, which means that the droplet impact could not be performed anymore. A particular pattern was made on the outer edge of the samples to identify and always keep the same surface orientation and also to assure that the micro-structured side is upwards.

At first, the samples were brushed with high purity nitrogen to remove small dust particles. Dirt particles must not be inside the gaps within the structure, since this can influence the impact morphology behavior. Then, the plasma treatments were performed in the cases that they are required. As stated, the "partner" sample receives exactly the same procedures as the structured one. Due to that, after the treatments, the apparent contact angle $\theta_{\text {app }}$ is measured on the "partner" sample. This angle was used to define the wetting behavior during the analysis. In future work, also the dynamic wetting behavior will be considered, however, in this study, the apparent contact angle is used to classify the experiments into four different wetting categories. An Optical Contour Analysis (OCA) system measured $\theta_{a p p}$ through the sessile drop method. Before starting the experiments, a thin film of isopropanol was spread at the top of the prism to allow the occurrence of total internal reflection for the bottom view inside the structured surface sample. The creation of bubbles was strictly avoided since they will create obscured areas on the bottom perspective. The imaging unit was set to start recording and the droplet dispensing system was initialized producing a homogeneous droplet chain. The droplet barrier is removed and a single droplet is allowed to enter the test section. In passing a LASER light barrier the imaging unit is triggered to record the impact. Finally, the surface samples have to be cleaned, and in case of plasma activation they have to be deactivated into a liquid "bath". Then, a rigorous cleaning procedure was implemented. After that, the experimental procedure can be repeated. Before increasing the impact velocity, every sample is checked at the optical microscope to investigate possible damages or irregularities on the micro-structures.

A wide range of impact conditions was tested. Table 2 shows the investigated parameter 
Table 2. Summary of the evaluated parameters. For each fluid, the indicated parameter range was investigated. The parameters that required a pre-treatment by plasma activation or polymerization are marked.

\begin{tabular}{|c|c|c|c|c|c|c|c|c|c|}
\hline \multicolumn{2}{|l|}{ Liquid } & \multicolumn{4}{|c|}{ Distilled water } & \multicolumn{4}{|c|}{ Isopropanol (2-propanol) } \\
\hline \multicolumn{2}{|l|}{ Material } & \multicolumn{4}{|c|}{$\operatorname{Lexan}^{\circledR}(P C)$} & \multicolumn{4}{|c|}{$\operatorname{Lexan}^{\circledR}(\mathrm{PC})$} \\
\hline \multicolumn{2}{|c|}{ Contact angle } & \multicolumn{4}{|c|}{$\begin{array}{c}0^{\circ *} \\
25^{\circ} \text { to } 40^{\circ *} \\
79.8^{\circ} \\
117^{\circ} \text { to } 122^{\circ}\end{array}$} & \multicolumn{4}{|c|}{$\begin{array}{c}0^{\circ} \\
66^{\circ} \text { to } 73^{\circ \star}\end{array}$} \\
\hline Impact & Re: & 4130 & 6860 & 9360 & 12240 & 1135 & 1745 & 2315 & 2955 \\
\hline Conditions & We: & 80 & 205 & 385 & 665 & 165 & 400 & 715 & 1165 \\
\hline \multicolumn{2}{|c|}{ Surface structure } & \multicolumn{4}{|c|}{$S 1, S 2, S 3, S 4$} & \multicolumn{4}{|c|}{$S 1, S 2, S 3, S 4$} \\
\hline \multicolumn{2}{|c|}{ Plasma treatments } & \multicolumn{8}{|c|}{ plasma activated $\left({ }^{*}\right)$ and plasma polymerized $\left({ }^{\star}\right)$} \\
\hline
\end{tabular}

space. Two different fluids were used to assess the influence of their thermophysical properties (density, surface tension and viscosity). Distilled water and isopropanol provided droplet diameters of $(2.3 \pm 0.1) \mathrm{mm}$ and $(2.3 \pm 0.1) \mathrm{mm}$, respectively. For distilled water, four wettability behaviors were studied from full wetting $\theta_{\text {app }} \approx 0^{\circ}$, highly wetting $\theta_{\text {app }} \in\left[25^{\circ} ; 40^{\circ}\right]$, partial wetting $\theta_{\text {app }}=79.8^{\circ}$ which is the natural contact angle between Lexan ${ }^{\circledR}$ and distilled water, to non-wetting $\theta_{\text {app }} \approx 120^{\circ}$. For isopropanol, only two wetting behaviors were possible since the natural contact angle with Lexan ${ }^{\circledR}$ already provided a full wetting condition and no further plasma activation can be applied. The samples were then treated with plasma polymerization and $\theta_{\text {app }} \in\left[66^{\circ} ; 73^{\circ}\right]$ was obtained. The impact velocity was increased four times through the droplet falling height providing four different impact energies for each fluid.

\section{Results and Discussion}

On the single droplet impact upon micro-structured surfaces with different surface patterns three impact regimes were identified: deposition, splashing and rebound. Figure 2 shows the regime maps for each surface pattern studied $(S 1-S 4)$ depending on the apparent contact angle and on the Weber number only for distilled water droplet impacts. For small Weber numbers, a decreasing wettability enhances the formation of partial rebound. Figure 3 shows the water droplet impact on hydrophobic $S 1$ surfaces from four different impact energies. Increasing impact energy, assumed to be the crucial parameter for the droplet dynamics, decreases the effect of surface wettability and inhibits the occurrence of partial rebound. Figure 3 (a) shows the formation of partial rebound from three different perspectives: lateral, top and bottom. Both, the scaling and the dimensionless time $\tau$ of each frame are presented. Here, $\tau=t u_{0} / D_{0}$, where $t$ is the time after impact. Due to the small adhesion between the surface and the liquid droplet a thick lamella is formed $(\tau=1.02)$ and spreads until reaching its maximum diameter $(\tau=3.39)$. Then, the lamella fastly recedes forming a high central jet that breaks producing partial rebound $(\tau=11.20)$. From the bottom perspective, it is noticeable that the liquid only penetrated the central region of impact and it spreads at the top of the structures without entering the gaps inside the micro-structures, the so-called heterogeneous wetting state $[7,12]$. This wetting condition was reached in all water experiments on partial wetting and non-wetting surfaces with only one exception. For low impact energies and $\theta_{\mathrm{app}}=79.8^{\circ}$, the liquid droplet homogeneously wets the $S 2$ surfaces. This is the surface pattern that requires the smallest pressure difference to reach a homogeneous wetting state since it presents the highest $b$ [7]. For the lowest impact energy, the outcome is always the same. However, increasing the impact energy, the individual influence of the surface pattern on the morphology stands out. Directional splashing is the dominant outcome of the impacts with $S 1$ and $S 2$, which have a similar 


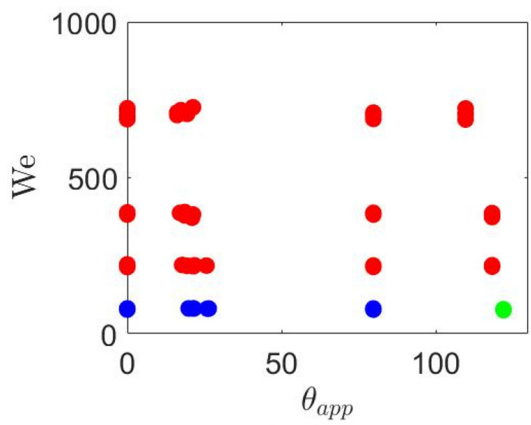

(S1)

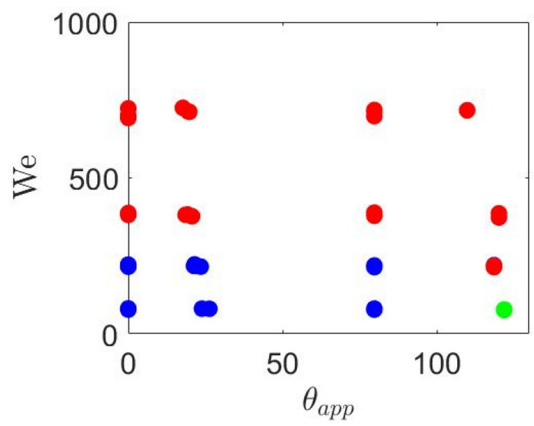

(S3)

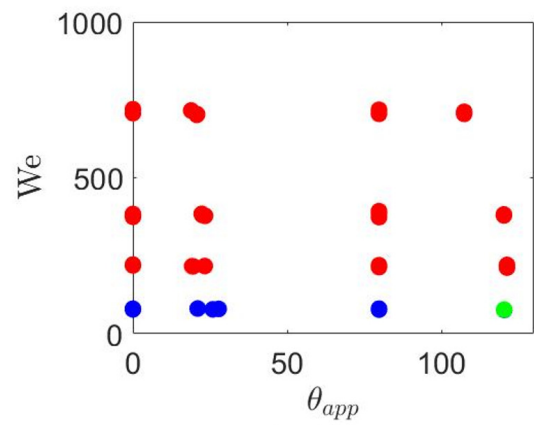

(S2)

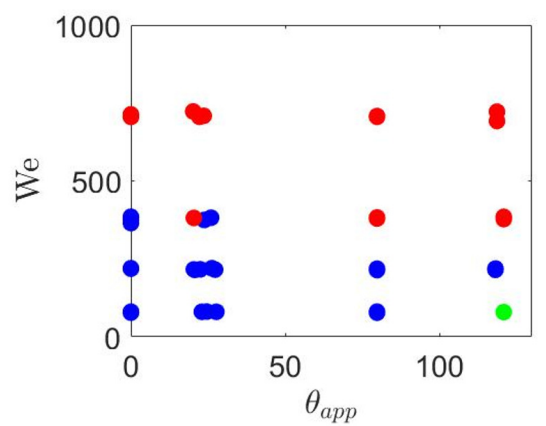

(S4)

Figure 2. Regime characterization of water droplet impact for each surface sample depending on the apparent contact angle $\left(\theta_{\text {app }}\right)$ and the Weber number $(W e)$.

(a) $\mathrm{We}=80$

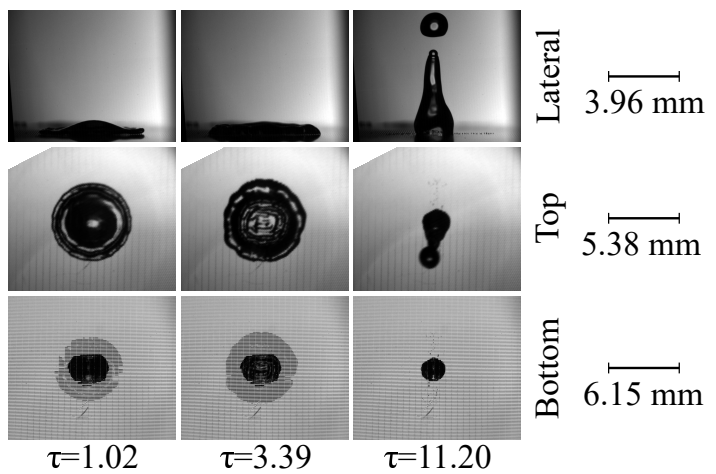

(c) $\mathrm{We}=385$

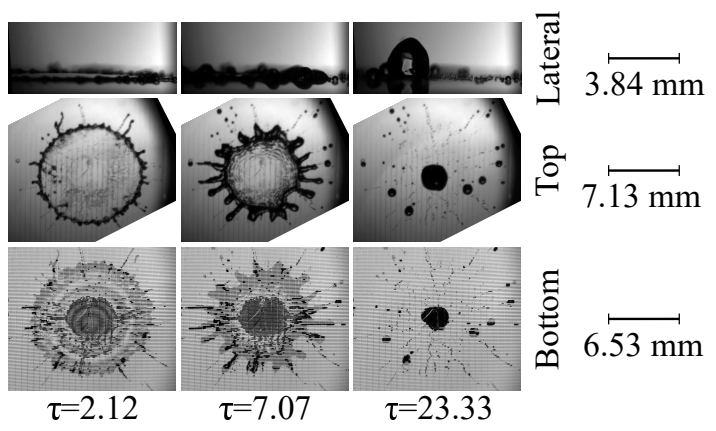

(b) $\mathrm{We}=205$

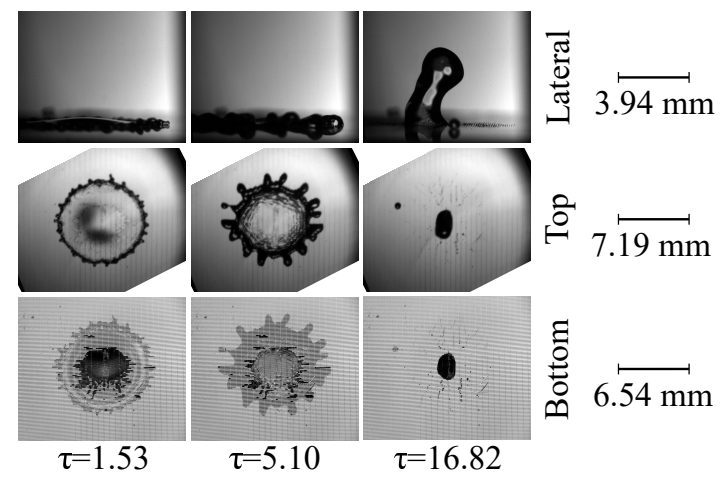

(d) $\mathrm{We}=665$

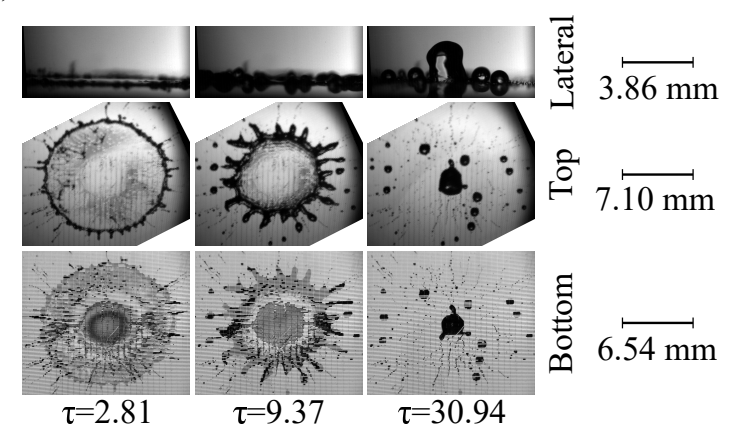

Figure 3. Water droplet impact upon hydrophobic $S 1$ surfaces for different impact energies: (a) $W e=80$; (b) $W e=205$; (c) $W e=385$; (d) $W e=665$. 
roughness factor $r$, independently of the wettability behavior. This parameter increases while decreasing the edge length of the micro-structures. Thus, small roughness factors $r$ seem to enhance directional prompt splashing.

From Figure 3 it is observable that the increment of impact energy promotes the formation of finger-like structures at the rim of the liquid lamella. Due to these liquid structures, the receding breakup is enhanced, since the fingers easily break at the surface discontinuities. In this way, the fluid that recedes inwards is not enough to form this high central jet. For even higher impact energies ( $W e=385$ and $W e=665$ ), the magnitude of finger formation increases and significant volumes of fluid are left on the surface during the receding phase. Wettability plays a minor role in the outcome determination since splashing was enhanced both, by the decrease in the roughness factor $r$ and by increasing the impact energy.

The impact of isopropanol droplets on surfaces with micro-structured patterns with different wettability behavior only originated deposition and splashing. Figure 4 (a) shows the regimes obtained depending on the Weber number $W e$ and the apparent contact angle $\theta_{\text {app }}$ for all surface structures. Directional splashing is observed even for low impact energies on $S 1$. The smaller interspaces between the rectangular grooves enlarge the possibility to develop prompt splash. Similar to water, for lower $W e$ and $\theta_{\text {app }} \in\left[66^{\circ} ; 73^{\circ}\right]$ only $S 2$ does not reach a heterogeneous wetting state. For isopropanol, non-wetted "holes" arise inside the liquid lamella, as shown in Figure 4 (b). At first, the bottom is not completely wetted by the fluid, $\tau=1.76$. Then, the fluid circumvents these areas creating spaces without any fluid, see top and bottom perspectives. Increasing the impact velocity, the role of surface topography continues to be the dominant influencing parameter since the transition to splash can be achieved by decreasing the surface roughness factor, $r$. However, increasing the impact energy even further seems to overlap the influence of surface topography since both, the phenomenon and their behavior, are similar for all micro-structured patterns.

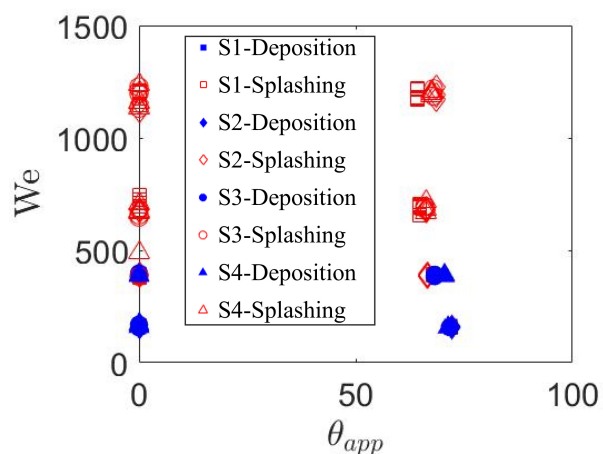

(a)

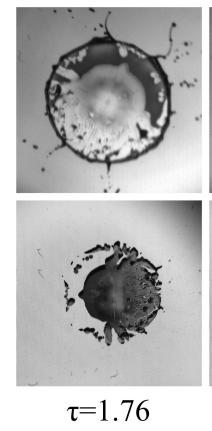

$\tau=1.76$

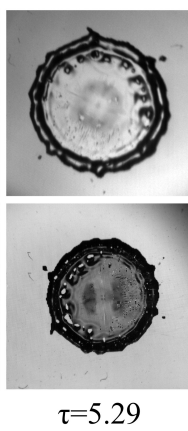

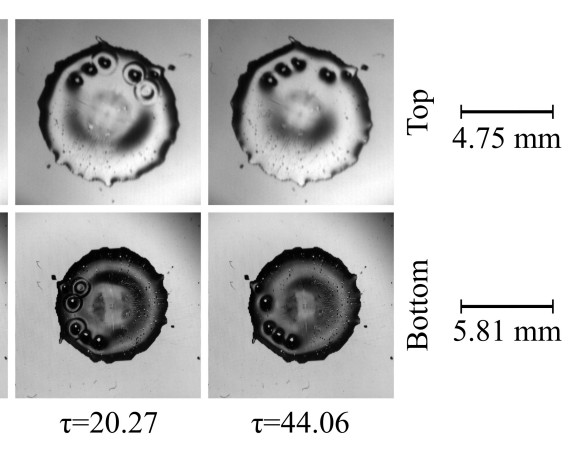

(b)

Figure 4. (a) Impact regimes of isopropanol droplets on micro-structured surfaces; (b) Heterogeneous wetting state for the isopropanol droplet impact onto partial wettable $S 3$ Lexan $^{\circledR}$ surfaces, $\theta_{\text {app }} \in\left[66^{\circ} ; 73^{\circ}\right]$, at $W e=715$.

\section{Conclusions}

In this study, surface topography and wettability behavior were combined in order to understand how these two parameters influence the impact morphology and outcome. Different fluids, wettability behaviors, micro-structured surface patterns and impact energies were tested and the deposition, splashing and rebound regimes were identified. In the water experiments, splashing was enhanced by surfaces with a low roughness factor $r$ and with increasing impact energy. At these impact energies, wettability only defines the spreading and receding behavior. Increasing impact energy, assumed to be the crucial factor in the droplet dynamics, decreases the effect of surface wettability inhibiting the occurrence of partial rebound. The heterogeneous wetting state is present on the impact with partially- and non-wetting surfaces. In the isopropanol experiments, the heterogeneous wetting state presents different results. Splashing is promoted by 
the smaller interspace between the rectangular grooves. For both fluids, increasing the impact energy even further overlaps the influence of surface topography.

\section{Acknowledgements}

The authors kindly acknowledge the financial support of this work by the Deutsche Forschungsgemeinschaft (DFG) in the frame of the International Research Training Group "Droplet Interaction Technologies" (GRK 2160: DROPIT) under the project number 270852890. Additionally, the authors would like to acknowledge the support of Fundação para a Ciência e a Tecnologia (FCT) through the Ph.D. scholarship SFRH/BD/140009/2018 and the project UIDB/50022/2020.

\section{Nomenclature}

$b$

$d, d_{1}, d_{2}$

$D_{0}$

$h$

$\mu$

$r$

$R_{a}$

$R e$

$\rho$

$\sigma$

$t, \tau$

$\theta_{\text {app }}, \theta_{Y}$

$u_{0}$

$w, w_{1}, w_{2}$

$W e$

Diagonal between two elements of the structure [m]

Distance between the micro-structured pillars [m]

Droplet diameter [m]

Height of the micro-structures [m]

Viscosity [Pa.s]

Roughness factor [-]

Arithmetical mean roughness [m]

Reynolds number $R e=\rho D_{0} u_{0} / \mu[-]$

Density $\left[\mathrm{m}^{3} / \mathrm{kg}\right]$

Surface tension $[\mathrm{N} / \mathrm{m}]$

Time and dimensionless time [s,-]

Contact angle (apparent, Young) [ $\left.{ }^{\circ}\right]$

Impact velocity [m/s]

Edge length of the micro-structures [m]

Weber number $W e=\rho u_{0}^{2} D_{0} / \sigma[-]$

\section{References}

[1] Yarin, A., Roisman, I., and Tropea, C., 2017, "Collision Phenomena in Liquids and Solids", Cambridge: Cambridge University Press.

[2] Zhang, M., Feng, S., Wang, L., and Zheng, Y., 2016, Biotribology, 5, pp. 31-43.

[3] Moita, A. S., and Moreira, A. L. N., July 13-17, 2003, 9th ICLASS - Europe (Institute for Liquid Atomization and Spray Systems).

[4] Pasandideh-Fard, M., Qiao, Y. M., Chandra, S., and Mostaghimi, J., 1996, Physics of Fluids, 8(3), pp. 650-659.

[5] Xu, L., 2007, Physical Review E, 75, pp. 056316.

[6] Tsai, P., van der Veen, R. C. A., van de Raa, M., and Lohse, D., 2010, Langmuir, 26(20, pp. 16090-16095.

[7] Hensel, R., Helbig, R., Aland, S., Braun, H., Voigt, A., Neinhuis, C., and Werner, C., 2013, Langmuir, 29, pp. 1100-1112.

[8] Bico, J., Thiele, U., and Quéré, D., 2002, Colloids and Surfaces A: Physicochemical and Engineering Aspects, 206, pp. 41-46.

[9] Foltyn, P., Restle, F., Wissmann, M., Hengsbach, S. and Weigand, B., 2021, Fluids, 6.

[10] Roisman, I. V., Lembach, A., and Tropea, C., 2015, Advances in Colloid and Interface Science, 222, pp. 615-621.

[11] Wensel, R. W., 1936, Industrial and Engineering Chemistry, 28, pp. 988-994.

[12] Mehdizadeh, N. Z., Chandra, S. and MOSTAGHIMI, J., 2004, Journal of Fluid Mechanics, 510, pp. 353-373. 